EUROPEAN ORGANIZATION FOR NUCLEAR RESEARCH

CERN SL-95-92 (AP)

\title{
Shielding Effects on Coherent Synchrotron Radiation *
}

\author{
S. A. Kheifetsł B. Zotter \\ CERN, CH 1211 Geneva 23
}

\begin{abstract}
A controversy concerning the shielding of coherent synchrotron radiation has existed for many years. Estimates of the reduction of radiated power by nearby conducting surfaces in LHC differ by several orders of magnitude when one uses the expressions given in two of the major papers in the field. A clarification of this problem as well as the expected values for coherent synchrotron radiation in LEP and LHC are presented here.
\end{abstract}

Geneva, Switzerland

*Paper presented at the Micro Bunches Workshop Sept.28-30, 1995, Brookhaven.

${ }^{\dagger}$ Permanent address: SLAC, Stanford, USA. 


\section{Introduction}

Practically all synchrotron radiation emitted by electrons in present circular accelerators or storage rings is incoherent, i.e. it is produced by each particle in a bunch independently. For high-energy machines, the maximum of the power spectrum lies at very short wavelengths, and many applications make use of this unique source of strong UV or X-ray radiation. However, the spectrum of the synchrotron radiation extends all the way down to small multiples of the revolution frequency. At such long wavelengths, the EM fields of different particles in a bunch can interfere constructively, and enhance the emitted radiation considerably. The power of this coherent radiation is proportional to the square of the number of particles per bunch.

The LHC will be the first proton machine with high enough energy to experience tangible effects of synchrotron radiation. A radiation damping time of the order of hours is expected, and the power hitting the vacuum chamber wall is no longer negligible. Since there will be a large number of protons per bunch, the question was raised whether there will be an appreciable enhancement of the usual incoherent synchrotron radiation by the coherent one.

In the standard literature, it was found that there exist a number of contradicting formulae for the shielding effect of the vacuum chamber walls. In particular, the pioneering work by Schiff in the 1940's[1], and the classical paper by Nodvick-Saxon[2], based on unpublished work by Schwinger [3], give reduction factors for the effect of nearby conducting walls which lead to results differing by several orders of magnitude for LHC.

Naturally, there are more recent publications which permit a correct estimate of this effect $[4,5]$, but here we want to explore the reasons for the existing discrepancy.

In addition to the coherent synchrotron radiation in the LHC - which, as expected, turned out to be utterly negligible, we are interested at CERN in specifying the conditions for observing it in LEP. In the near future, enough $\mathrm{SC}$ cavities will be installed there for operation at higher energy (LEP2) that it should be possible to reach submillimeter bunch lengths at injection. Together with the operation of strong wigglers with small bending radius this should make its observation possible in the next few years. Amongst other uses, it could become a valuable tool for beam diagnostics.

\section{LHC Parameters}

We start by calculating the power radiated in LHC by incoherent synchrotron radiation. With maximum magnetic fields of about $8 \mathrm{~T}$ in the $\mathrm{SC}$ magnets, protons with an energy of up to $\mathcal{E}=7 \mathrm{TeV}(\gamma=7460)$ can be kept at the bending radius $\rho=2.70 \mathrm{~km}$, fitting into the LEP tunnel with a circumference of 
$26.7 \mathrm{~km}$ (revolution frequency $f_{0}=\omega_{0} / 2 \pi=11.24 \mathrm{kHz}$ ). In the present design, there will be $k_{b}=2835$ bunches of over $N_{b}=10^{11}$ protons in each beam in order to reach the desired high luminosity.

We assume from the start $\beta=1$, which is always quite well fulfilled in highenergy electron storage rings. The incoherent radiation power can be found with an expression which was first derived by Schott[6]

$$
P_{\text {tot }}=\frac{2}{3} \frac{k_{b} N_{b} r_{p} \mathcal{E}_{0} \omega_{0}}{\rho} \gamma^{4}
$$

where $r_{0}=1.537 \cdot 10^{-18} \mathrm{~m}$ is the classical radius of the proton, and its rest energy is $\mathcal{E}=1.503 \cdot 10^{-10} \mathrm{~J}$. With these values one obtains a radiated power of about

$$
P_{\text {inc }}=3.67 \mathrm{~kW},
$$

corresponding to $216 \mathrm{~mW} / \mathrm{m}$ which has to be absorbed largely by the cryogenic system. Any substantial enhancement would thus be rather expensive in terms of cooling power.

In spite of the rather long bunches $(\sigma=7.5 \mathrm{~cm})$, the simple expression for the power of coherent synchrotron radiation gives indeed much higher values. We calculate directly the ratio of coherent to incoherent power, given by Ref.[1]:

$$
\frac{P_{\text {coh }}}{P_{\text {inc }}}=\frac{3^{7 / 6}}{4 \pi} \Gamma\left(\frac{2}{3}\right) \frac{N_{b}}{\gamma^{4}}\left(\frac{\rho}{\sigma}\right)^{4 / 3}
$$

Substituting the values of the parameters of the LHC results in the surprising fact that its coherent power would be more than 20 times higher than the incoherent one, and this would present an excessive load on the vacuum chamber and the cryogenics, unless a considerable reduction occurs by the shielding effects of the vacuum chamber walls.

\section{Reduction of Coherent Synchrotron Radiation by Shielding}

Particles radiate coherently at a wavelength $\lambda$ if their relative distances are smaller than half this wavelength. The EM fields of such particles interfere constructively, and the resulting field is the same as the field radiated by one particle with the total charge equal to the sum of all charges. If the characteristic length of the longitudinal charge distribution is $\sigma_{s}$, then the condition for coherence of the radiation in free space is simply

$$
\sigma_{s} \leq \lambda / 2 \pi
$$

Unlike the case of incoherent radiation, for which the radiation power is proportional to the number $N_{b}$ of particles in a bunch, the power of coherent radiation 
is proportional to $N_{b}^{2}$. To understand how this comes about, consider two particles moving in a magnetic field on a circular trajectory. Their positions have azimuthal angles $\phi_{1}$ and $\phi_{2}$ with respect to some arbitrary azimuth taken as zero. Correspondingly, their currents have the phase factors $\exp \left(-i \phi_{1}\right)$ and $\exp \left(-i \phi_{2}\right)$. The $n$-th harmonics of the fields excited by the particles have therefore the same phase factors $\exp \left(-i n \phi_{1}\right)$ and $\exp \left(-i n \phi_{2}\right)$.

To find the total field created by all $N_{b}$ particles of a bunch, we need to sum their individual fields taking into account the corresponding phase factors. For the radiation power averaged over the longitudinal particle distribution $S(\phi)$ we find

$$
P_{n}\left|\sum_{k}^{N} e^{-i n \phi_{k}}\right|^{2}=N P_{n}+N(N-1) P_{n} f_{n},
$$

where $P_{n}$ is the incoherent power emitted by each particle, and the form factor for coherent radiation $f_{n}$ is given by

$$
f_{n}=\left[\int d \phi \cos (n \phi) S(\phi)\right]^{2}
$$

For example, for a Gaussian distribution with rms bunch length $\sigma_{s}$

$$
\begin{gathered}
S(\phi)=\frac{\rho}{\sqrt{2 \pi} \sigma_{s}} \exp \left(-\frac{\phi^{2} \rho^{2}}{2 \sigma_{s}^{2}}\right), \\
f_{n}=\exp \left(-\frac{n^{2} \sigma_{s}^{2}}{\rho^{2}}\right) .
\end{gathered}
$$

Since $\lambda \approx 2 \pi \rho / n$, the coherence condition $\mathrm{Eq}$. (3) follows more formally from these equations.

The first term on the right hand side of Eq. (4) gives the power of incoherent radiation, which is proportional to the number of particles in a bunch or to its current, and independent of the bunch shape. The second term gives the power of coherent radiation, which for $N_{b} \gg 1$ is proportional to the square of the number of particles, equivalent to the square of the bunch current $I_{n}$ at the $n$-th harmonic. Such a dependence makes it suitable to describe coherent radiation by an effective impedance $Z_{n}$ in accordance with Ohm's law $P_{n}=Z_{n} I_{n}^{2}$.

For all practical particle distributions, the condition Eq. (3) limits coherent synchrotron radiation to microwave or far-infrared frequencies. Hence, the wavelengths of the radiated power are usually comparable to the transverse size of the vacuum chamber and/or to the distance to poles of a magnet. Under these conditions, the coherent radiation and its characteristics can be changed substantially. When the walls are conducting, induced charges will decrease the EM fields created directly by the bunches. Magnetic materials have a similar effect due to induced currents. This phenomenon is referred to as shielding and is the stronger, the closer the induced charges. From this consideration it is clear that the shielding must depend on the size of the vacuum chamber or the distance to the magnet poles. 


\section{Coherence and Shielding Lengths}

To take into account the presence of conducting walls, and to evaluate the shielding effect for coherent radiation, we need to modify the coherence condition Eq. (3).

Consider, for example, a case of two parallel conducting plates placed above and below the plane of motion at $z= \pm h / 2$. The tangential components of the electric field should satisfy the proper boundary conditions on the plates. For perfectly conducting plates these components should be zero there. That means that the EM field must contain factors which make the appropriate components of the field vanish on the plates. Hence, the radiation field then has to be expanded in a double series containing two integer summation indices:

the radial harmonic number $n$, and

the vertical harmonic number $p$.

Accordingly, the wave vector $\mathrm{k},|k|=\omega / \mathrm{c}$ of the EM field acquires a vertical component $k_{z}$ which cannot be smaller then $\pi / h$ - the lowest value of the vertical wave number which is consistent with the boundary condition. As we discussed previously, the opening angle $\alpha$ into which most of the radiation is emitted, is smaller than $n^{-1 / 3}$, which means that $\pi / h<k \alpha=\omega \alpha / c=n \alpha / \rho<n^{2 / 3} / \rho$. Here $\rho$ is the local radius of curvature of the trajectory due to the magnetic field. Hence, only harmonics with $n>(\pi \rho / h)^{3 / 2}$ can be emitted coherently. The more exact condition is $[7]$

$$
n>n_{t h} \equiv \sqrt{\frac{2}{3}}\left(\frac{\pi \rho}{h}\right)^{3 / 2} .
$$

Usually $\rho$ is much bigger then $h$. Then radiation can be emitted coherently only at rather high harmonics.

Thus the condition of coherence in the presence of conducting plates is more stringent than the one in free space. We rewrite Eq. (3) as

$$
n<n_{c}=\rho / \sigma
$$

to get approximately

$$
\frac{\rho}{\sigma_{s}}>n>\sqrt{\frac{2}{3}}\left(\frac{\pi \rho}{h}\right)^{3 / 2} .
$$

The power spectrum of coherent radiation increases toward shorter wavelengths. However, this increase is limited by the bunch length since the radiation ceases to be coherent when the wavelength becomes smaller then the bunch length. The bunch length needs to be smaller than the chamber half height over $\pi$, multiplied with a usually quite small factor

$$
\sigma<\frac{h}{\pi} \sqrt{\frac{3 h}{2 \pi \rho}}
$$

or the emission of coherent radiation will be suppressed. 


\section{$5 \quad$ Shielding by Two Plates}

We now turn to the effect of shielding. We first consider the case when a charge moves between two perfectly conducting plates, positioned at $z= \pm h / 2$ above and below the plane of the orbit[3],[2].

The $n$-th harmonics of the EM field of a point charge moving in the mid-plane between the plates can be found from a Green function $G_{n}\left(r, \phi, z ; r^{\prime}, \phi^{\prime}, z^{\prime}\right)$ which vanishes at $z= \pm h / 2[8]$

$$
G_{n}=\frac{i}{2 h} \sum_{p=1}^{\infty} \sum_{m=-\infty}^{\infty} \sin p \pi\left(\frac{z}{h}+\frac{1}{2}\right) \sin p \pi\left(\frac{z^{\prime}}{h}+\frac{1}{2}\right) e^{i m\left(\phi-\phi^{\prime}\right)} G_{r}\left(r, r^{\prime}\right),
$$

where

$$
G_{r}\left(r, r^{\prime}\right)=J_{m}\left(\gamma_{p} r\right) H_{m}^{(1)}\left(\gamma_{p} r^{\prime}\right) \quad \text { for } \quad r<r^{\prime} .
$$

Here $\gamma_{p}=\sqrt{(n / \rho)^{2}-(p \pi / h)^{2}}$, and we get the expression for $r>r^{\prime}$ simply by interchanging the arguments of the Bessel functions.

The power of radiation at the $n$th harmonic is

$$
P_{n}=\operatorname{Re}\left\{4 i n r_{0} \mathcal{E}_{0} \omega_{0} \int_{-\pi}^{\pi} d\left(\phi-\phi^{\prime}\right) G_{n}\left(\rho, \phi, 0 ; \rho, \phi^{\prime}, 0\right)\left[1-\cos \left(\phi-\phi^{\prime}\right)\right] e^{-i n\left(\phi-\phi^{\prime}\right)}\right\} .
$$

After performing the integration over the angles one gets

$$
P_{n}=\frac{4 \pi n r_{0} \mathcal{E}_{0} \omega_{0}}{h} \sum_{p=1,3, \ldots}^{p<n h / \pi \rho}\left(J_{n}^{\prime 2}+\frac{(p \pi \rho / h)^{2}}{n^{2}-(p \pi \rho / h)^{2}} J_{n}^{2}\right),
$$

where the argument of the Bessel functions is $\gamma_{p} \rho$.

Only propagating modes with the numbers $p<n h / \pi \rho$ contribute to the energy loss, since for evanescent modes the product $J_{m} H_{m}^{(1)}$ is purely imaginary.

\section{Spectrum of Coherent Synchrotron Radiation}

In the ultra-relativistic case, and when the condition $\pi \rho / h \gg 1$ is fulfilled, most of the radiation is produced at the sufficiently large harmonic numbers $n$. That makes the following asymptotic expansions of the Bessel functions valid:

$$
J_{n}\left(\gamma_{p} \rho\right)=\frac{1}{\sqrt{3} \pi}\left(\frac{g}{n}\right)^{2} K_{2 / 3}\left(\frac{g^{3}}{3 n^{2}}\right)
$$

and

$$
J_{n}^{\prime}\left(\gamma_{p} \rho\right)=\frac{1}{\sqrt{3} \pi}\left(\frac{g}{n}\right) K_{1 / 3}\left(\frac{g^{3}}{3 n^{2}}\right)
$$

where $g=p \pi \rho / h$. 
Since the modified Bessel functions $K_{\nu}(x)$ become exponentially small for large $x$, only harmonics with $n>g^{3 / 2}$ contribute substantially to the sum in Eq. (15). Hence, $n \gg p \pi \rho / h$ and the second term in Eq. (15) can be simplified. The result for a bunch of $\mathrm{N}$ particles is $[3,2]$

$$
P_{n}=\frac{N^{2} r_{0} \mathcal{E}_{0} \omega_{0}}{\rho} \frac{4 \rho}{3 \pi h} \sum_{p=1,3, \ldots}^{p<n h / \pi \rho} \frac{g^{4}}{n^{3}}\left[K_{1 / 3}^{2}(x)+K_{2 / 3}^{2}(x)\right],
$$

where the argument of the modified Bessel functions is $x=g^{3} / 3 n^{2}$.

The main contribution to the sum in Eq. (18) is produced by the region in which the argument of the modified Bessel functions is small. The condition $x<1$ for $p=1$ is the same as the one given in Eq. (10). When $n_{t h} \gg 1$ (see definition in Eq. (8), the sum in Eq. (18) can be replaced by an integral over $x$. Hence,

$$
P_{n}=\frac{2 C}{3^{1 / 3} \pi^{2}} \frac{N^{2} r_{0} \mathcal{E}_{0} \omega_{0}}{\rho} n^{1 / 3} \text { for } n_{t h}<n<n_{c}
$$

where[9]

$$
C=\int_{0}^{\infty} d x x^{2 / 3}\left[K_{2 / 3}^{2}(x)+K_{1 / 3}^{2}(x)\right] \approx 3.68 .
$$

One can see that the spectrum of coherent radiation is essentially the same as that of incoherent radiation. The extra factor $N_{b}$ increases the power by a large amount, making the bunch moving in a magnetic field a very powerful source of the electro-magnetic radiation. Formula Eq. (19) defines the radiation spectrum for $n_{t h}<n$.

In the range $n<n_{t h}$, we need to evaluate the sum in Eq. (18) for $x>1$. For this we use the asymptotic expansion of the functions $K_{\nu}$ for $\nu=1 / 3$ and $2 / 3$, which decrease exponentially with increasing $x$. Hence it is enough to keep only the first term in the sum over $p$ and we get

$$
P_{n}=\frac{2^{5 / 3}}{\pi} \frac{N^{2} r_{0} \mathcal{E}_{0} \omega_{0}}{\rho} n^{1 / 3}\left(\frac{n_{t h}^{2}}{2 n^{2}}\right)^{2} \exp \left(-\frac{n_{t h}^{2}}{2 n^{2}}\right) \quad \text { for } \quad n<n_{t h},
$$

which gives the shape of the spectrum below the threshold harmonic.

\section{Total Power of Coherent Synchrotron Radiation}

To obtain the total power of coherent synchrotron radiation, the power spectrum Eq. (19), should be multiplied by the spectrum of the bunch distribution and summed over all allowed harmonic numbers $n$.

For a particular case of the Gaussian distribution, the particle density spectrum is given by formulae in Eq. (7):

$$
P_{c o h} \approx \frac{N^{2} r_{0} \mathcal{E}_{0} \omega_{0}}{\rho} \sum_{n_{t h}}^{n_{c}} n^{1 / 3} e^{-\left(\frac{n \sigma_{s}}{\rho}\right)^{2}} .
$$


Since the harmonic numbers $n$ which produce the main contribution to the sum in this equation are very large, the summation can be replaced by an integration over $x=\left(n \sigma_{s} / \rho\right)^{2}$ :

$$
P_{c o h} \approx \frac{N^{2} r_{0} \mathcal{E}_{0} \omega_{0}}{2 \rho}\left(\frac{\rho}{\sigma_{s}}\right)^{4 / 3} F\left(x_{t h}\right)
$$

where the form factor is

$$
F\left(x_{t h}\right)=\int_{x_{t h}}^{x_{c}} d x x^{-1 / 3} e^{-x}
$$

and the limits of integration are

$$
x_{t h} \equiv \frac{2 \pi^{3} \rho \sigma_{s}^{2}}{3 h^{3}} \quad, \quad x_{c}=4 \pi^{2} .
$$

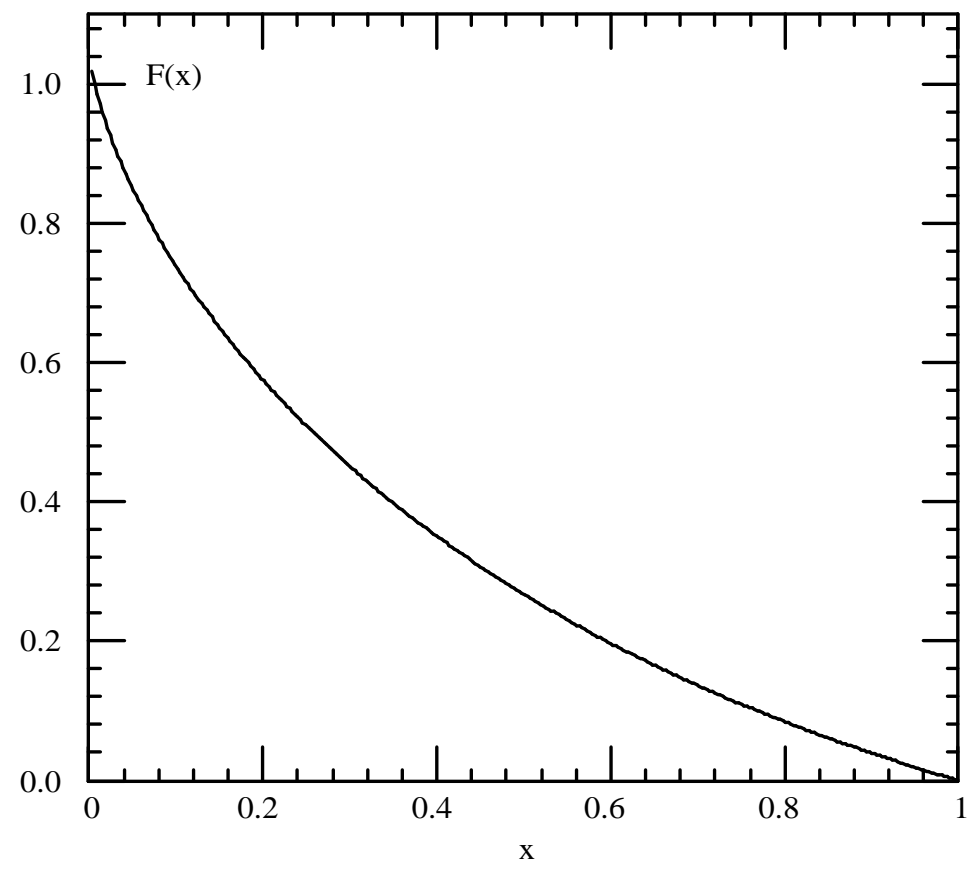

Figure 1: Function $F(x)-F(1)$, cf. Eq. (24)

It can be expressed as a difference between two incomplete $\Gamma$-functions of order $2 / 3$ :

$$
F\left(x_{t h}\right)=\Gamma\left(2 / 3, x_{t h}\right)-\Gamma\left(2 / 3,4 \pi^{2}\right) \approx \Gamma\left(2 / 3, x_{t h}\right) .
$$

and it vanishes when $x_{t h} \geq 4 \pi^{2}$. The function shown in Fig. 1 is actually $F(x)=$ $\Gamma(2 / 3, x)-\Gamma(2 / 3,1)$, and one has to add $\Gamma(2 / 3,1) \approx 0.303$ to get $F(x)$.

For some values of its argument, estimates can be obtained by using appropriate expansions of $F(x)$. 
1. When the argument is very small, $x_{t h} \ll 1$, then $F(x) \approx \Gamma(2 / 3) \approx 1.354$. The power of coherent radiation is given by Eq. (23) with this value of $F\left(x_{t h}\right)$, in agreement with that derived originally by Schiff[1].

2. When $1<x<4 \pi^{2}$, the function $F\left(x_{t h}\right) \approx x^{-1 / 3} e^{-x}$. Except for the exponential - which is usually a very small factor, Eq. (23) then agrees with the expression derived by Nodvick-Saxon[2].

3. When $x \geq 4 \pi^{2}$, there is no more coherent radiation.

Similar results could be obtained for other particle distributions, e.g. for a uniform distribution in the range $-\sigma_{s}<s<\sigma_{s}$. The density spectrum of such a distribution is $f_{n}=[\sin x / x]^{2}$, where $x=n \sigma_{s} / \rho$. However, its spectrum is much wider as it does not fall off exponentially as a Gaussian. The results for the uniform distribution can be derived in the same manner as shown above, which differs from that of Ref.[2] mainly by the fact that the summation over $p$ is replaced by an integral, rather than that over $n$, and that the summation over $n$ is restricted to the allowed range of mode numbers $n_{t} h<n<n_{c}$. By extending the limits of the summation, the result is still an upper limit but a rather crude one. This fact was not taken into account in the paper[2], and the estimate for the total power of coherent radiation may therefore become a very large upper limit.

However, the main difference between our result - essentially in agreement with a several published papers on this subject - and the expression from Ref.[2] is due to their use of an unrealistic uniform distribution, with no strong rolloff at high frequencies as for more physical distributions like a Gaussian. Their expression agrees with Eq. (23) if $F\left(x_{t h}\right)$ is simply replaced by $x_{t h}^{-1 / 3}$.

\section{Estimates for Existing Storage Rings}

The total power of coherent synchrotron radiation is mainly determined by the single parameter $x_{t h}$ Eq. (25). It depends on two different physical quantities:

(a) shielding, characterized by the ratio $\rho / h$, and

(b) bunching, characterized by the ratio $\sigma / \rho$.

In all existing electron storage rings for a high particle energy, $x_{t h} \gg 1$ and coherent radiation is very week at normal operating conditions. In order to be able to observe this type of radiation in storage rings, they have to be operated with very short bunches, fulfilling Eq. (11), unless they are specially designed to limit the magnitude of the parameter $x_{t h} \approx 1$. This can be achieved by choosing a small ratio $\rho / h$, i.e. a small radius of curvature and a large chamber diameter. In storage rings with large bending radius, the radius of curvature can be reduced by locally high magnetic fields, such as wigglers or undulators.

The ratio of coherent radiation power to incoherent can be obtained from 
Eqs.(4) and (29) to become:

$$
\frac{P_{c o h}}{P_{\text {inc }}}=\frac{N}{2 \gamma^{4}}\left(\frac{\rho}{\sigma_{s}}\right)^{4 / 3} F\left(x_{t h}\right) .
$$

In LEP, a large number of superconducting RF cavities are presently being installed for operation at energies of up to $90 \mathrm{GeV} /$ beam. Some 192 cavities should be ready by the end 1996 , with 64 cavities more later. The total installed RF voltage will increase from the present $300 \mathrm{MV}$ to over $3 \mathrm{GV}$. At the same time, low-emittance lattices with lower momentum compaction are being developed to maximize luminosity at the higher operating energies. At present the shortest (theoretical) bunch length is about $2 \mathrm{~mm}$. With a more than 10 -fold increase of RF voltage, and a reduction of $\alpha$ by another factor 2 , one should be able to reach bunch lengths well below $0.5 \mathrm{~mm}$. Using the existing wigglers with a minimum bending radius of $\rho=50 \mathrm{~m}$ in a vacuum chamber of $3.5 \mathrm{~cm}$ half height, one finds $x_{t h}=6.03$ for $\sigma=0.5 \mathrm{~mm}$. Then $F\left(x_{t h}\right) \approx 1.3310^{-3}$. With $10^{11}$ particles $/$ bunch - to keep bunch lengthening small - the unshielded coherent radiation would yield some $2.9 \mathrm{~kW}$, but only about $4 \mathrm{~W}$ when shielding is taken into account. This power will be further reduced because of the short length of the wiggler magnets, which are somewhat shorter than the formation length of the radiation at these wavelengths. The bending magnets in LEP have a large bending radius of $\rho=$ $3.1 \mathrm{~km}, x_{t h}=230$, and coherent radiation will be completely suppressed unless the bunch length can be further reduced to below $0.2 \mathrm{~mm}$.

Similarly, concerns about possible effects of coherent synchrotron radiation in the LHC, presently under construction at CERN, are completely unfounded. While the LHC will be the first proton storage ring where incoherent synchrotron radiation has tangible effects, the large bending radius and the bunch length of over $70 \mathrm{~mm}$ yield an enormous parameter $x_{t h}=1.710^{6}$, and all coherent radiation will be completely suppressed.

However, construction of a dedicated storage ring for coherent synchrotron radiation with short bunches and small bending radii in large vacuum chambers appears entirely feasible and has actually already been proposed in Ref.[10].

\section{Conclusions}

The emission of coherent synchrotron radiation by high energy charged particles traveling on circular orbits is reviewed. Several important aspects of the radiation, such as coherence, bunching, and shielding, are considered. A long-standing discrepancy in the estimates of power reduction by shielding is explained and removed. The reduction of the power of coherent synchrotron radiation by shielding is expressed in terms of a single parameter $x_{t h}$.

In existing storage rings, practically no coherent radiation can be expected in their normal mode of operation. In the large electron-positron storage ring LEP 
at CERN bunches need to be shortened to well below $1 \mathrm{~mm}$ rms to see its effects. This should soon become possible with the high RF voltage being installed for operation as LEP2 at energies up to $90 \mathrm{GeV} /$ beam. Concerns about the effects of coherent radiation in the large proton storage ring LHC, now under construction, are dispelled.

\section{Acknowledgments}

We would like to thank F. Ruggiero for raising the problem of coherent synchrotron radiation in $\mathrm{LHC}$, and $\mathrm{L}$. Rivkin for inciting one of the authors (SK) to work on the problem of conflicting expressions for power reduction by shielding. as well as A. Hofmann for evaluating an integral used in the derivations.

\section{References}

[1] L.I. Schiff, Rev. Sci. Instr.17, 6-14, (1946).

[2] J. S. Nodvick and D.S. Saxon, Phys. Rev. 96, 180-184 (1954).

[3] J. Schwinger, "On Radiation by Electrons in a Betatron", unpublished, (1945).

[4] R. Warnock, "Shielded Coherent Synchrotron Radiation" SLAC-Pub-5523 (May 1991).

[5] S. Kheifets, B.Zotter, CERN Divisional Report SL 95-43 (May 1995) and S. Kheifets, CLIC-Note 287 (July 1995).

[6] G. Schott, Annalen der Physik 24, 635-660 (1907).

[7] S. Heifets, A. Michailichenko, "On the Impedance due to Synchrotron Radiation", SLAC Note AP-83 (1990), also Proceedings of the Particle Accelerator Conference, San Francisco 1991, p.458.

[8] P. M. Morse and H. Feshbach, "Methods of Theoretical Physics", McGrawHill, New York, 1953.

[9] A. Hofmann, private communication (1994).

[10] J.B. Murphy, et al, "Millimeter Wave Coherent Synchrotron Radiation in a Compact Electron Storage Ring", to be published in Proc. of 1995 Nat. Conf. on High-Energy Accel., Dallas, 1995. 